Neuroscience and Biobehavioural Reviews, 2006, 30(3): 273-275

\title{
Relationship between the brain and aggression
}

\section{J. Martin Ramirez}

I am both privileged and humbled to have the opportunity to contribute to the excellence of this journal, as editor of this special issue on relationships between the brain and aggression. It has been possible by the work of numerous scientists and scholars. I wouldl ike to start the preface thus showing my deep appreciation to them. Robert Adamec and Verity Brown encouraged me to put it together. It further benefited from the thoughtful comments of anonymous reviewers. For the editing and proofreading, I am also indebted to VerityBrown whose keen eyesight, dedication, and professionalism buoyed me when I started to worry because English is not my native language. I have also been most fortunate to receive the superb technical assistance of Elsevier professionals, such as Ms Purdy, Slattery, andJeeves. And, finally, I proudly acknowledge my wife Tina for giving me the support I handed, not only emotional but also practical, in its edition. In those respects I feel she was my co-editor.

There are two main reasons to have special issues in a journal like NBR: scientific and educational. First, and most obviously, these issues include theoretical and review articles that help put in perspective what is known about a particular area of research. Having a set of papers dealing with a coherent topic helps visualizing new avenues for research. Second, these special issues would make a great material for discussion in graduate-level seminars on neuroscience and in aggression research. This present special issueis as cientific fruit of one of a series of CICAs (International Colloquia on Conflict and Aggression) held during the last two decades (20 meetings all over the world: the first met in Seville in 1983, and subsequently other meetings have taken place in different countries in Europe, Americas, Africa, and Asia), with 14 scientific books and special issues published on the different aspects which have been addressed. A characteristic of these meetings is their international, interdisciplinary, comprehensive approach, in the understanding that most major developments arise in zones where disciplines mesh. Any discipline that becomes completelycutoff from what is going on inother areas will probably endup like the geographically isolated species in the Galapagos islands and eventually fizzle out and disappear. This approach provides a useful biosocial focus, bringing together a number 
of disciplines to study more effectively the complex relationship between the brain and aggression, with the particular goal of bridging the persistent gulf between biologically based approaches and those built on the traditional social sciences, in the understanding that real solutions will only be found through the integration of work from many different levels and fields.

The19th CICA meeting, held at Rhodes (Greece), 14 -17 September 2004, was a good platform for bringing together scientists working animal models related to aggression with scientists studying similar processes in people. Thirty-eight scientists from 19 countries attended, and 17 papers were presented, grouped in the following five symposia: neurogenetics of aggression; social conflict, stress, coping and psychopathology; brain asymmetry and aggression; conflict resolution; and terrorist weapons. Those nine articles closer to neuroscience have been selected for this special issue. Even if they cover a vast territory, touching on various disciplines, there is an interactive approach dealing with a type of behaviour in which links with the brain are rather difficult to define and measure. They may be grouped in three rather consistent blocks. The first three articles are related to a repertoire of aggression and other close constructs in normal, psychopathological, and experimental situations. The second three articles focus on neural, endocrinal, and neurogenetic mechanisms of aggression. And the last three articles deal with the relationship between stress and aggression in humanand non-human primates. The issue starts with a paper in which Ramirez and Andreu examine the important issue of the relations between main types of aggression, on the one hand, and other related psychological variables, such as anger, hostility, and impulsivity, on the other. The manuscript offers some theoretical considerations on the concept of human aggression and its main types, and provides a mini-review of the empirical results of a research on the topic, still in progress, done along the last two decades by their group on aggression research at the UniversidadComplutense Madrid. Its content should be of interest to researchers and clinicians alike.

Whereas the first paper is concerned with normal subjects, the second article, the product of joint Hungarian-Dutch research, is a lucid review and presentation of intriguing ideas about causes of abnormal aggression in humans and how to model them. The Haller-Kruk paper addresses two very important issues: the development of animal models of pathological aggression in humans, and the relation between adaptive and pathological aggression in animals and humans. They present data suggesting that there is a mutual positive short lasting feedback between the adrenocortical 
stress response and a brain mechanism involved in the control of aggression at the hypothalamic-amygdala level. They also develop a rationale for animal models of pathological aggression that are rooted in deviation from adaptive aggression. While very preliminary, the ideas regarding novel ways to model human aggression disorders in rodents by paying attention more to the nature (targeting, behavioral controls), rather than simply quantity, of attacks maybe a potentially valuable insight. The paperofAdamec'sCanadian group, at Newfoundland University, concerns the lasting consequences on brain and behaviour of another kind of aggression, the predatory attack. In an elegantly written paper, theyfocus onthe victim, or target, of aggression. It is an excellent account on the lasting neurochemical and electrophysiological changes induced in rodents following a single cat-exposure. The experiments involved sophisticated technology and the results are interesting. The authors convincingly show that both kinds ofchanges are highly predicted bythe behaviour of the cat and bythe response of the rat, and they conclude that there are longlasting changes in brain function and behaviour, the latter servingasamodel for post-traumatic stress disorder. In few words, it is an excellent contribution to our understanding of trauma. Adams' reviewis the updated of an already classical motivational systems model proposed by him more than a quarter of century ago to explain the brain mechanisms of aggressive behaviour. It is a scholarly review of the potential neural mechanisms of the different kinds of agonistic behaviours, offense and defense, as well as of patrol/marking, also related to aggressive behaviour. Attention is also given to the neurallocation of learning and hormonal effects. It is one of the best, concise and highly valuable reviews on brain mechanisms of aggressive behaviour, and it should be very useful to those outside and inside the field of aggression research.

After writing an excellent paper on the empirical findings regarding the association between testosterone and human aggressive behaviour, Archer presents an interesting review of the evidence accumulated in humans about a number of predictions within the theoretical framework of the challenge hypothesis, originally applied to account for testosterone behaviour relationships in birds. Along this extensive manuscript, the author, president of the International Society for Research onAggression, revises more or less proximate evidence about the predictions he previously established, sometimes employing statistical tests, others by anexhaustive narration of studies. Most of the predictions were supported by the review of current research. The current manuscript will advance the literature but 
presenting a unique theoretical approach to interpreting findings in behavioural endocrinology.

The paper presented bythe Nelson sgroup, at OhioState University, is a very nice piece of work related to neurogenetics of aggression, with an upto-date review of the role of nitricoxide in the regulation of aggressive behaviour. Theydescribe how male mice with targeted deletion of the genes encoding the neuronal isoform of nitricoxidesynthase display more aggressive behaviour than wild-type mice. This specific elevated aggression reflects some pleiotropic effects of these genes: an increased sensitivity to painful stimuli, which may prolong aggressive interactions, a lower fearful or 'anxiety-like' behaviour, anda lower 'depressive-like' one. Lastly,the multiple, and often unanticipated, effects of targeted gene disruption on aggressive behaviour are also considered. Elizabeth Susman has an excellent and long standing reputation as a student of the relationship between stress and aggression. The purpose of her paper was to present a theoretical perspective on the development of persistent antisocial behaviour, emphasizing stress and early brain development. The attenuation of endocrine physiology of the stress systemis considered a key mechanism involved in persistent antisocial behaviour. The paper stresses a very important topic, the ideasl ntegrated in the theory are sound, the relevant data in the field are presented, and the end result is a valuable contribution to the literature. The two final reviews are product of a joint effort $b$ yHoness andMarin, scientists from Oxford and Madrid, respectively. Their manuscripts are an exhaustive review of the literature on stress and aggression, and their interactions, in non-human primates, predominantly from the genus Macaca. One paper is focused to the behavioural and physiological aspects, whereas the other addresses the environmental influence on both fields. The authors have done an excellent job not only of summarizing the current state of knowledge in these fields but also integrating them in a concise and engaging way, in spite of the complex and often contradictory nature of their relationships. I believe these both papers present avaluable contribution to the literature contribution to the field.

One of the main goals of neuroscience is to understand how the brain, a not-very-attractive three-pound organ, but with a staggering complexity (a hundred billion neurons connected by a hundred trillion synapses largely shaped bythe genes in normal prenatal development, even if also sculpted by information coming in from the senses) allows the mind to be mended and manipulated. The largely innate geometry, cabling, and gross features of the brain can have real consequencesfor thinking, feeling, andbehaving. 
Obviously, noone doubts that learning takes advantage of the plasticity of thebrain. But we are not indefinitelymalleable byexperience; we are not just products of our environments. The influence of the psychosocial environment cannot be disentangled from the biology. The changes take place within a matrix of genetically organized structure. Genes providea developmental potential, but it can be activated only in conjunction with the environment. All the potential for thinking, learning, feeling, and behaving that distinguishes human species from other animal species lies in the information contained in the DNA of the fertilized ovum. In few words, genes are part of, but not all of the story. And aggression Is no exception. The variety of articles in this issue has shown, had we had not known it yet, that aggression, far from being a unitary process, is a complex phenomenon composed of intertwined processes, result of complex interactions among several physiological, motivational, and behavioural systems, with contributions from the social as well as the physical environment. Some individuals are constitutionally more prone to violence than others, and they have a distinctive personality profile -they tend to be impulsive, low in intelligence, hyperactive and attention-deficient (which usually emerges in earlychildhood, and persists through the lifespan). For example, convicted murderers and other violent, antisocial people, in spite of growing up perhaps surrounded by a rather negative environmental atmosphere, are likelyto have limbic systems enmeshed with a smaller and less active prefrontal cortex, the part of the brain that governs decision-making and inhibits impulses. But the fact that we all have an innate capacity to be aggressive does not implythat we must be aggressive, since factors other than biology are always involved in its development. In fewwords, even if aggression is influenced by biology, it is not determined by genetic information,but it is also modulated by many environmental factors. A basic understanding of aggression and of its multiple causes therefore is necessary not only to propose positive remedies, but also to understand humanity better, because one needs not accept aggression as an inevitable fate. And this fact gives one hope! 